was later. We were discussing the X-ray determination in my laboratory shortly before the War broke out.

It is not, of course, necessary that the most closely packed planes should be the glide planes in all cases, although the most closely packed direction is always the glide direction. This emerges very clearly from some work which I carried out with Dr. Y. S. Chow ${ }^{1}$ on body-centred cubic crystals. The matter of the glide plane must, therefore, remain open until I, or someone else, put it to the X-ray test.

E. N. DA C. Andrade.

${ }^{3}$ Andrade, E. N. da C., and Chow, Y. S., Proc. Roy. Soc., A, 175, 290 (1940)

\section{Photograph of a Nuclear Disintegration in a Wilson Chamber}

THE presence of heavy ionization tracks has often been observed in Wilson chamber photographs; those due to the emission of $\alpha$-particles from radioactive impurities can be easily eliminated. The rest are due to nuclear disintegrations produced by cosmic rays. Usually they emerge from lead plates placed inside such chambers. Photographs of disintegrations originating in the gaseous volume of a Wilson chamber are rare; so far as is known to us, Anderson and Neddermeyer ${ }^{1}$ have photographed one, and Zlotowsky ${ }^{2}$ another. The particles are supposed to be produced by a nuclear evaporation process leading to the emission of both protons and neutrons ; in some cases $\alpha$-particles have also keen detected amongst them.

The accompanying photograph shows the emission of four ionizing particles from a nucleus in the gaseous volume of a Wilson chamker filled with oxygen. From the density of ionization along their tracks, the particles appear to be heavier than $\alpha$-particles and are multiple-charged. As in Anderson's photograph ${ }^{\mathbf{I}}$, the emitted particles are confined within a hemisphere, and conservation of momentum requires that either a number of neutrons have been emitted in the opposite direction or that a fast non-ionizing particle is responsible for the disintegration-the latter assumption appears more reasonable. A faint mesotron track is seen to cross the lead plate $(2 \cdot 2 \mathrm{~cm}$. thick) and follow the continuation of track 4 ; it cannot be taken to be responsible for the process of disintegration, as the track in question does not show any scattering or change in ionization density after passing the point of origin of the disintegrated nucleus. Tracks 1 and 4 are of a similar nature; both show jagged outlines, due to ionization by recoil ions produced by nuclear collisions with the primary charged particles; the ionization density along the track of the particle diminishes. Such ionization tracks are to be expected of slow multiple-charged heavy particles, the effective charge of which diminishes with velocity, due to successive neutralizations. Such tracks hare been observed in Wilson chamber photographs of fission of the uranium nucleus ${ }^{3}$.

Tracks 2 and 3 are broad and of uniform width throughout their whole lengths, except near the very end where there is a sharp fall of ionization-due to partial neutralization of charges on the ionizing particles. These tracks are similar to those due to $\alpha$-particles except they are broader; they may be due to doubly-charged heavy particles. The end portions of their tracks are crossed transversely by

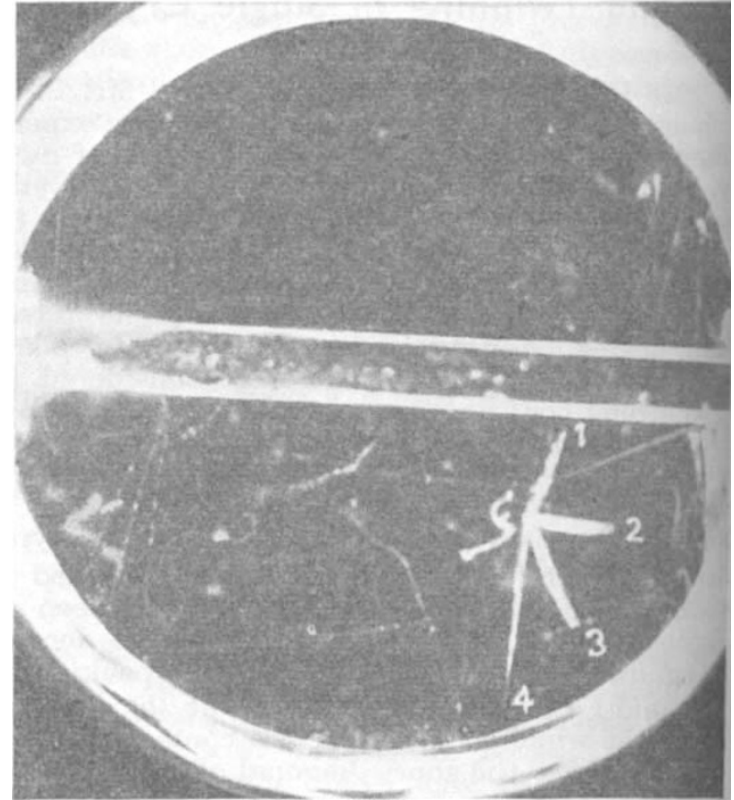

THE RANGES OF TRACKS $1,2,3$ AND 4 CONVERTED TO NORMAL PRESSURES ARE $5 \cdot 33,4 \cdot 76,6 \cdot 56$ AXD 9.01 CM. RESPECTIVEIY.

thin ionization tracks. An obvious explanation of the observed effect is that, due to pure chance, the heavy-particle tracks are crossed at corresponding end points by two electron tracks. But the simultaneous occurrence of such events must be extremely rare. An alternative explanation, which appears to us plausible, is that each of the particles producing the tracks 2 and 3 emits, after it has slowed down, a pair of ionizing particles. In the original photograph, the track crossing the end portion of 3 is slightly bent towards the latter, showing that the particles emitted had a velocity component in the direction of motion of the parent particle. If this explanation is accepted, we have further to account for the fact that the pair of particles are emitted in diametrically opposite directions. This can be accounted for on the assumption that the original nucleus disintegrates into two simpler nuclei which move in opposite directions. Among the lighter nuclei, it is known that ${ }^{8} \mathrm{~B}$ produced by the interaction ${ }^{7} \mathrm{Li}+{ }^{1} \mathrm{H}$ disintegrates with the emission of a pair of $\alpha$-particles, the Wilson chamber photographs of which have been obtained by Dee and Walton ${ }^{4}$. The tracks of the pair emitted from the ends of tracks 2 and 3 appear to be too thin to be due to $\alpha$-particles.

The other alternative is that a pair of (oppositely charged) electrons were emitted from the nuclei of the primary particles. I am not aware that emission of a pair of electrons from an excited nucleus in diametrically opposite directions has been previously observed, and there may be some theoretical difficulty in accounting for such an occurrence.

The interpretation of the origin of the tracks in the photograph given in this communication has arisen out of a discussion with Prof. D. M. Bose, to whom my thanks are due.

M. SINHA.

Bose Institute,

Calcutta. July 1 .

1 Anderson and Neddermeyer, Phys, Rev., 50, 268 (1936).

2 Zlotowsky, Nature, 140, 585 (1937).

${ }^{3}$ Brostrom, Boggild, Lauritsen, Phys. Rev., 58, 651 (1940), et seq.

${ }^{4}$ Dee and Walton, Proc. Roy. Soc., 141, 733 (1933). 